\title{
Nailfold videocapillaroscopy and serum VEGF levels in scleroderma are associated with internal organ involvement
}

\author{
Maria De Santis ${ }^{1}$ Angela Ceribelli ${ }^{1,2} \cdot$ Francesca Cavaciocchi $^{1} \cdot$ Chiara Crotti $^{1}$ \\ Marco Massarotti ${ }^{1}$ - Laura Belloli ${ }^{1,3}$ - Bianca Marasini ${ }^{1}$ - Natasa Isailovic ${ }^{1}$. \\ Elena Generali ${ }^{1} \cdot$ Carlo Selmi $^{1,2}$
}

Received: 11 January 2016/ Accepted: 23 January 2016/Published online: 15 February 2016

(C) The Author(s) 2016

\begin{abstract}
Purpose Nailfold videocapillaroscopy (NVC) identifies the microvascular hallmarks of systemic sclerosis (SSc) and vascular endothelial growth factor (VEGF) and may play a pivotal role in the associated vasculopathy. The aim of the present study was to compare NVC alterations with clinical subsets, internal organ involvement, and serum VEGF levels in a cohort of selected SSc cases.

Methods We studied 44 patients with SSc who were evaluated within 3 months from enrollment by NVC, skin score, severity index, pulmonary function tests, carbon monoxide diffusing capacity (DLCO), echocardiography, pulmonary high-resolution computed tomography (HRCT), gastroesophageal (GE) endoscopy or manometry or X-ray, and serum autoantibodies. Serum VEGF-A levels were determined by ELISA in $72 \mathrm{SSc}$ patients and 31 healthy controls.

Results Giant capillaries were inversely correlated with age $(p=0.034, r=-0.34)$ and to the extent of reticular pattern at HRCT $(p=0.04, r=-0.5)$. Avascular areas were directly correlated with capillaroscopy skin ulcer risk index (CSURI) $(p=0.006, r=+0.4)$ and severity index $(p=0.004, r=+0.5)$. The mean capillary density was directly correlated to the ulcer number $(p=0.02$, $r=+0.4)$ and to DLCO/alveolar volume $(p=0.02$, $r=+0.4)$ and inversely correlated with severity index $(p=0.01, r=-0.4)$ and skin score $(p=0.02, r=-0.4)$.
\end{abstract}

Carlo Selmi

carlo.selmi@unimi.it

1 Rheumatology and Clinical Immunology, Humanitas Research Hospital, Rozzano, Milan, Italy

2 BIOMETRA Department, University of Milan, Milan, Italy

3 Present Address: Division of Rheumatology, Niguarda Hospital, Milan, Italy
Serum VEGF levels were higher in the SSc population vs controls $(p=0.03)$ and inversely correlated with DLCO $(p=0.01, r=-0.4)$ and directly with ground-glass and reticular pattern at HRCT ( $p=0.04, r=+0.4$ for both). Conclusions Our data suggest the importance of NVC not only for the diagnosis, but also for the global evaluation of SSc patients. Of note, serum VEGF levels may act as a biomarker of interstitial lung involvement.

Keywords Autoimmunity - Biomarkers - Personalized medicine $\cdot$ Systemic sclerosis $\cdot$ Endothelium

\section{Introduction}

Systemic sclerosis (SSc) is an immune-mediated vascular and fibrotic systemic disease [1], with striking female predominance and with unknown pathogenesis involving both genetics and environmental factors [2-5]. Microvascular alterations underlying Raynaud's phenomenon and rearrangement of the capillary network characterize the early phases of the disease. Nailfold videocapillaroscopy (NVC) can easily identify SSc microvascular hallmarks as giant capillaries and avascular areas, but also less specific and frequent changes as ectasia, microhemorrhages, bushy capillaries, and reduced capillary density. NVC is now recognized as an indispensable tool for the early diagnosis of SSc together with the presence of Raynaud's phenomenon and specific serum autoantibodies such as anticentromere and anti-topoisomerase I (anti-Scl70) [6]. The NVC scleroderma pattern has been recently included in the last SSc classification criteria [7], but the role of NVC during the course of SSc is less defined. NVC alterations can change with disease progression and severity, and Cutolo et al. have reported three different NVC 
scleroderma patterns, i.e., early (presence of giant capillaries), active (prevalence of giant capillaries), and late pattern (prevalence of avascular areas) [8], possibly mirroring the different phases of the disease. More recently, Sebastiani et al., developed a score associated with ulcer development called capillaroscopy skin ulcer risk index (CSURI > 2.94) [9], thus suggesting a role for NVC other than the early diagnosis of SSc.

While the pathogenetic mechanisms in SSc remain elusive, the vascular endothelial growth factor (VEGF) is one of the main mediators of angiogenesis and vasculogenesis, being involved in SSc vasculopathy. Whether and which changes in VEGF and its receptor levels are associated with SSc and how they contribute to scleroderma vasculopathy continue to be unclear [10-15].

The aim of this study is to investigate the correlations between NVC alterations, Cutolo's NVC patterns and CSURI with the fine clinical SSc phenotype including internal organ involvement, SSc severity score [16], and serum VEGF levels in a cohort of well-defined SSc cases.

\section{Patients and methods}

\section{Subjects}

We enrolled 44 patients fulfilling the 2013 ACR/EULAR criteria for SSc [7] without other overlapping rheumatic diseases and for whom the following data were available: NVC images of the right and left II-V fingers; all clinical, immunologic, clinimetric, and instrumental evaluations (Table 1); and one serum sample for each SSc patient. All SSc cases were evaluated between 2001 and 2014, but serum samples, clinical, and instrumental data analyzed in the study were obtained within 3 months from NVC. The characteristics of the study population are summarized in Table 1 . Serum samples of 31 female healthy subjects (median age 65 years, range 3-80 years) and additional 32 women with SSc (median age 67 years, range 40-78; median disease duration 8 years, range 1-17 years; diffuse SSc 10; ACA (+) 17 cases; anti-Scl70 (+) 8 cases) were also obtained and used as controls. All subjects enrolled signed a written informed consent for serum samples and data analysis after approval from the local ethical committee.

\section{NVC analysis}

Two independent readers (MDS and CC), blinded to patient information, analyzed and recorded the mean capillary density per finger and the total numbers of giant capillaries, neoangiogenesis, and avascular areas on four consecutive NVC fields extending over $1 \mathrm{~mm}$, in the middle of the nailfold of the right and left second, third,
Table 1 Characteristics of the SSc cohort $(n=44)$

\begin{tabular}{ll}
\hline & SSc $(n=44)$ \\
\hline F (\%) vs M (\%) & $42(95.4 \%)$ vs $2(4.6 \%)$ \\
Median age, yy(range) & $66(34-80)$ \\
Median disease duration, yy (range) & $9(1-16)$ \\
D (\%) vs L (\%) & $10(22.7 \%)$ vs $34(77.3 \%)$ \\
anti-Scl70 (+) (\%) & $12(27.3 \%)$ \\
ACA (+) $(\%)$ & $22(50 \%)$ \\
Others Abs $(\%)^{a}$ & $10(22.7 \%)$ \\
Iloprost $(\%)$ & $25(56.8 \%)$ \\
Bosentan $(\%)$ & $7(15.9 \%)$ \\
Ulcers $(\%)$ & $15(34.1 \%)$ \\
Early NVC pattern $(\%)$ & $6(13.6 \%)$ \\
Active NVC pattern $(\%)$ & $20(45.4 \%)$ \\
Late NVC pattern $(\%)$ & $18(40.9 \%)$ \\
GE disease $(\%)$ & $36(81.8 \%)$ \\
sPAP $>45$ mmHg $(\%)$ & $3(6.8 \%)$ \\
DLCO <50 \% (\%) & $7(15.9 \%)$ \\
DLCO/AV <50 \% (\%) & $1(2.3 \%)$ \\
FVC <80 \% (\%) & $8(18.2 \%)$ \\
Honeycombing lung $(\%)$ & $4(9.1 \%)$ \\
Rodnan skin score $>14(\%)$ & $9(20.4 \%)$ \\
\hline ACA &
\end{tabular}

$A C A$ anti-centromere, anti-Scl70 anti-topoisomerase, $A V$ alveolar volume, $D$ diffuse, $D L C O$ carbon monoxide diffusing capacity, $F$ female, $F V C$ forced vital capacity, $G E$ gastro-esophageal, $L$ limited, $M$ male, $N V C$ nailfold videocapillaroscopy, $s P A P$ systolic pulmonary artery pressure on echocardiography, $S S c$ systemic sclerosis, $v s$ versus

a 1/10 anti-Ku, 2/10 anti-RNApol III, 1/10 anti-Th/To

fourth, and fifth fingers; early, active, and late scleroderma patterns, as described by Cutolo et al. [8], and CSURI as described by Sebastiani et al. [9] were assessed.

\section{Clinical and imaging assessment}

Age, disease duration (from the first non-Raynaud symptom), cutaneous subset, modified Rodnan skin score (mRSS), digital ulcers (current), SSc severity index [16], and treatments received were analyzed. All patients underwent echocardiography for pulmonary hypertension screening with systolic pulmonary artery pressure (sPAP) assessment ( 3 patients had PAPs $>45 \mathrm{mmHg}$ related to interstitial lung disease); pulmonary function tests for forced vital capacity (FVC) and carbon monoxide diffusing capacity (DLCO) and DLCO/alveolar volume (DLCO/AV) assessment; high-resolution computed tomography of the lung (HRCT) with the assessment of ground-glass reticular pattern and honeycombing extent as described elsewhere [17-19]. Gastro-esophageal (GE) involvement was investigated during history and assessed in symptomatic patients with esophageal manometry or X-ray or endoscopy. 


\section{Laboratory tests}

Serum was obtained from the peripheral blood of the $44 \mathrm{SSc}$ patients included in the study, 32 unselected SSc patients, and 31 sex- and age-matched healthy controls. Laboratory tests for SSc cases included complete blood cell count, C-reactive protein (CRP) level, serum creatinine concentration, urine analysis, complement $\mathrm{C} 3$ and $\mathrm{C} 4$, ACA (immunofluorescence on Hep2 cells), anti-Scl70 (ELISA), protein immunoprecipitation (IP) of K562 cell extract radiolabeled with ${ }^{35} \mathrm{~S}$-methionine and by RNA-IP through silver staining; specificities of autoantibodies were determined using specific reference sera [20, 21] for serum antibody assessment.

VEGF-A was measured by quantitative sandwich enzyme-linked immunosorbent assay (ELISA) (Quantikine kits; R\&D). Concentrations were calculated using a standard curve generated with specific standards provided by the manufacturer.

\section{Statistical analysis}

All data are presented as median and range for continuous variables. The two-sample Mann-Whitney's test and contingency tables were used as appropriate. The Spearman's test was used for correlations. $P$ values $<0.05$ were considered to be significant.

\section{Results}

Our analysis was performed on 44 SSc cases, 32 unselected SSc patients, and 31 sex- and age-matched healthy controls. The number of giant capillaries was inversely correlated with age ( $p=0.034, r=-0.34$; Fig. 1a) and to the extent of reticular pattern on HRCT ( $p=0.04, r=-0.5$, Fig. 1b). The number of giant capillaries was higher in patients without serum anti-Scl70 or ACA and in those with skin score $>14$ ( $p=$ ns; Table 2$)$. As expected, the number of giant capillaries was significantly higher in patients with active NVC pattern ( $p<0.0001$; Table 2).

The degree of neoangiogenesis was higher in SSc patients with GE involvement, honeycombing, DLCO $<50 \%$, sPAP $>45 \mathrm{mmHg}$, higher VEGF levels (Table 2), and in SSc patients treated with bosentan ( $p=0.016$; Table 2).

The number of avascular areas was directly correlated to severity index ( $p=0.004, r=+0.5$; Fig. 2a) and inversely to DLCO/AV ( $p=0.02, r=-0.4$; Fig. $2 \mathrm{~b}$ ). Moreover, the number of avascular areas was higher in SSc patients with anti-Scl70 or ACA ( $p=$ ns; Table 2$)$ and in those with higher sPAP ( $p=0.03$; Table 2$)$ or needing iloprost $(p=0.04$; Table 2 ) or bosentan ( $p=$ ns; Table 2 ) for digital ulcers. As expected, the number of avascular areas was significantly higher in patients with late NVC pattern $(p<0.0001$; Table 2) and CSURI > 2.94 ( $p=0.01$; Table 2$)$.

The mean capillary density was directly correlated with the number of digital ulcers $(p=0.02, r=+0.4)$ and to DLCO/AV ( $p=0.02, r=+0.4)$, and inversely with the severity index $(p=0.01, r=-0.4$; Fig. 3a) and skin score ( $p=0.02, r=-0.4$; Fig. 3b). Moreover, the mean capillary density was lower in patients with anti-Scl70, skin score $>14$, $(p=\mathrm{ns}$; Table 2$)$, and, as expected, in patients with late NVC pattern ( $p=0.0007$; Table 2$)$ and CSURI $<2.94$ ( $p=0.047$; Table 2 ). The highest mean capillary density was found in patients with early NVC pattern ( $p=0.005$; Table 2).

Cutolo's NVC late pattern was significantly more prevalent in SSc with higher sPAP ( $p=0.025$; Table 3$)$ and honeycombing $(p=0.037$; Table 3$)$, in patients receiving iloprost $(p=0.039$; Table 3$)$ or bosentan ( $p=0.017$; Table 3 ) for digital ulcers, and in those with the lowest VEGF levels ( $p=0.02$; Table 3 ). Moreover, the late pattern was significantly less frequent in patients with CSURI $<2.94$ ( $p=0.02$; Table 3 ).

CSURI directly correlated wih the number of avascular areas $(p=0.006, r=+0.4)$ and was higher in patients with late NVC pattern $(p=0.02$; Table 3$)$.
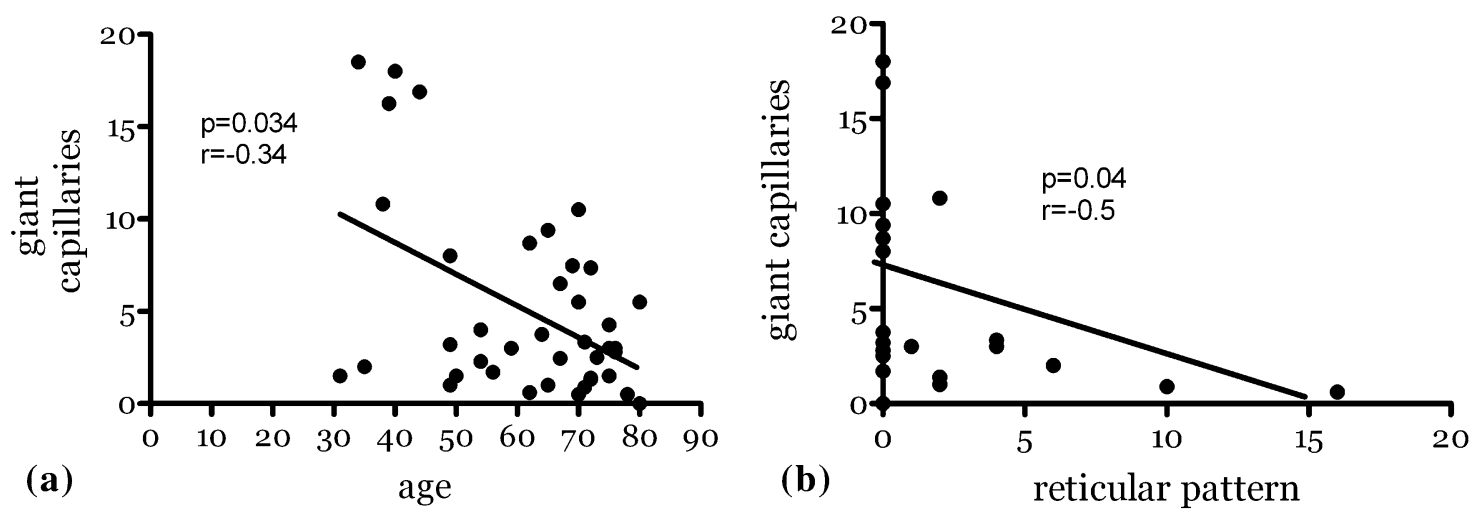

Fig. 1 Giant capillaries inversely correlated with age and reticular pattern on high-resolution computed tomography 


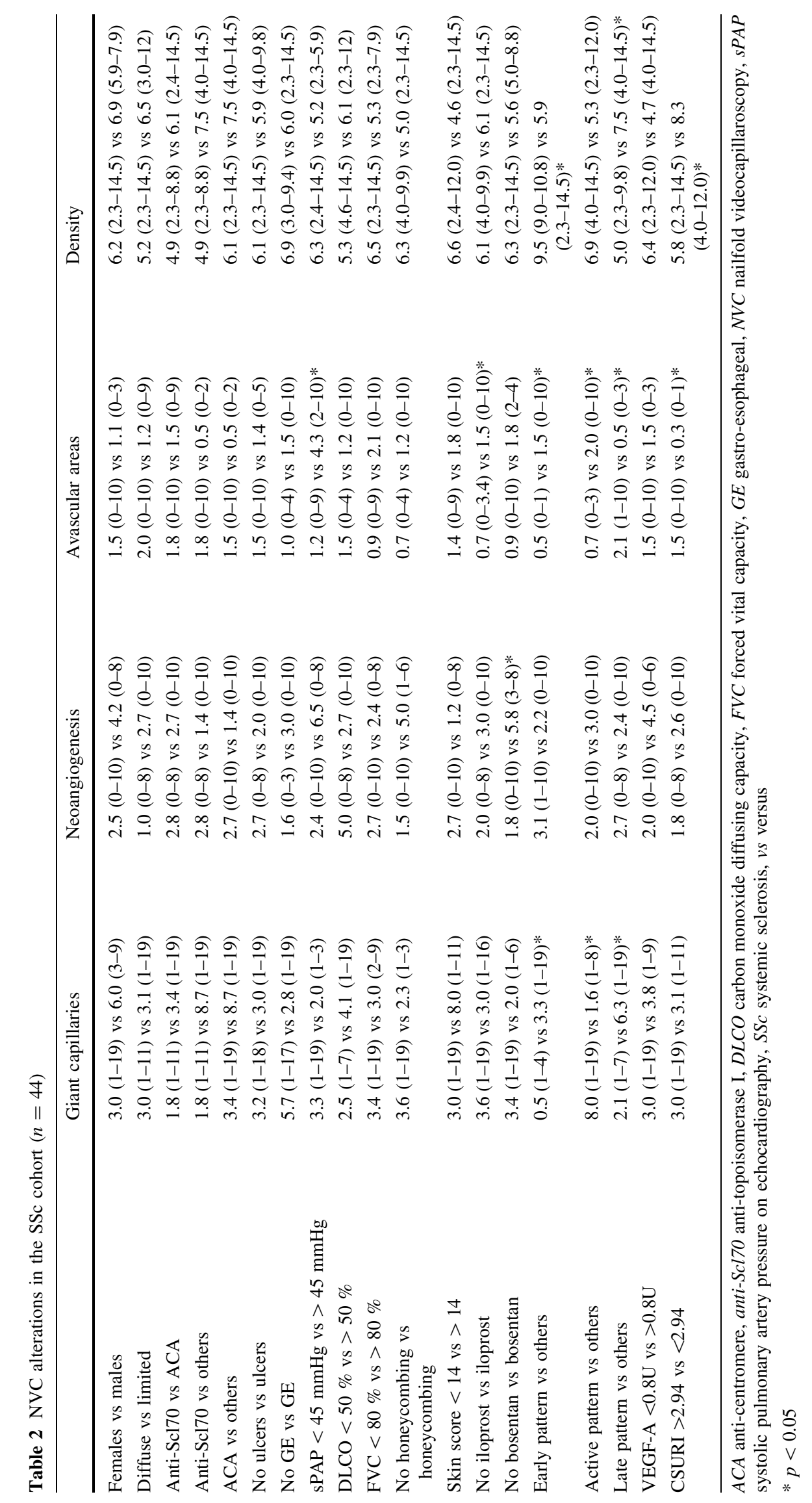



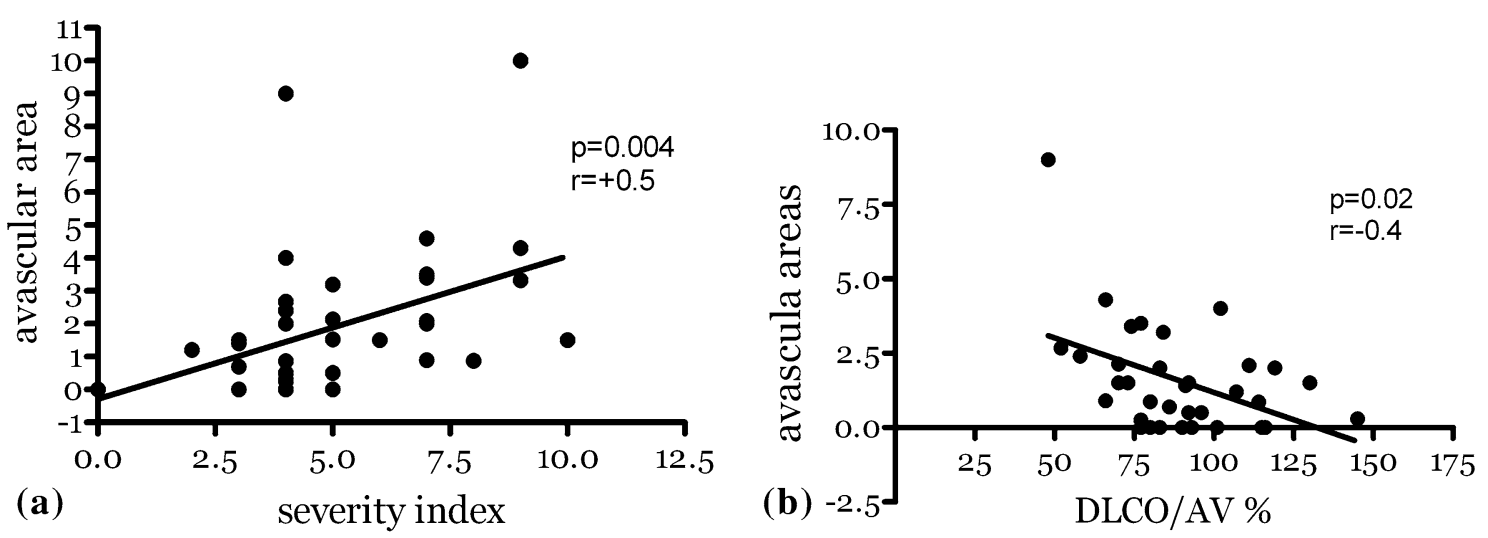

Fig. 2 Avascular areas directly correlated with severity index and inversely with DLCO/AV
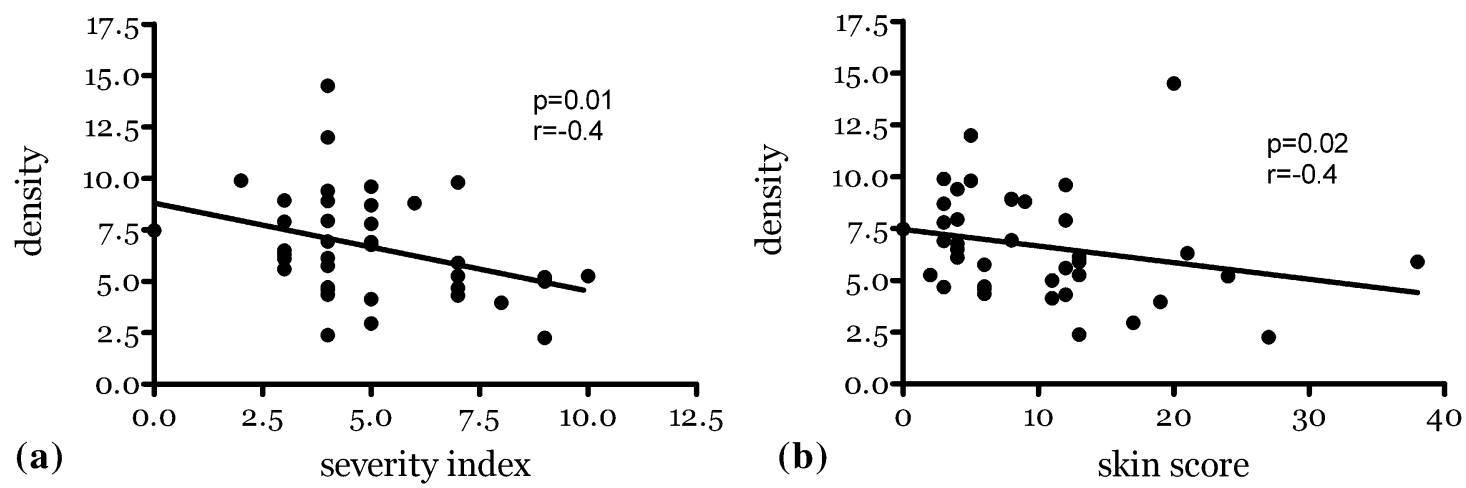

Fig. 3 Mean capillary density correlated inversely with severity index e with skin score

Serum VEGF-A was higher in SSc vs healthy controls (median 0.5, range 0.02-3.05 vs median 0.4, range 0.2-0.5, respectively; $p=0.03$ ). Among SSc cases, VEGF-A was inversely correlated with DLCO $(p=0.01, r=-0.4$; Fig. 4b), and directly with ground-glass and reticular pattern extent on HRCT $(p=0.04, r=+0.4$ for both; Fig. 4b). Moreover, VEGF-A levels were lower in the serum of SSc patients with evidence of interstitial lung disease (median 0.39 , range $0.1-3.05$ vs median 0.48 , range $0.14-1.70$, respectively; $p=0.44)$.

\section{Discussion}

The hallmarks of the NVC scleroderma pattern include giant capillaries and avascular areas, albeit poorly specific for SSc, as they are also observed in other connective tissue diseases characterized by Raynaud's phenomenon or interstitial lung disease such as dermatomyositis and mixed connective tissue disease [22]. Nevertheless, almost all scleroderma patients had one or both of those alterations, while the association of scleroderma pattern to other connective tissue diseases is not quite clear, especially for rare diseases as anti-synthetase syndrome [23, 24]. The present study was aimed at a clear definition of the NVC patterns and fine phenotyping in well-defined SSc clinical subtypes in association with serum VEGF-A levels. Our data cumulatively support the finding that NVC may be helpful not only for the diagnosis, but also for the global and longitudinal evaluation of patients with SSc. Further, serum VEGF-A levels may represent a biomarker to predict interstitial lung involvement in SSc.

In our cohort, the crude prevalence of giant capillaries and avascular areas appears to be related to the severity of the disease as indicated by the correlations with interstitial lung disease, SPAP, higher skin score, or SSc severity index. When confirmed in larger cohorts, these data may suggest a helpful role of this simple counting score as marker of internal organ involvement. Moreover, it would be interesting to test it in cases of SSc sine scleroderma or in other connective tissue diseases with NVC scleroderma pattern and risk of interstitial lung disease or pulmonary arterial hypertension. Another interesting point is to explore NVC in SSc patients with rare autoantibodies, since in our cohort SSc patients with rare autoantibodies seem to have more giant capillaries compared to avascular 


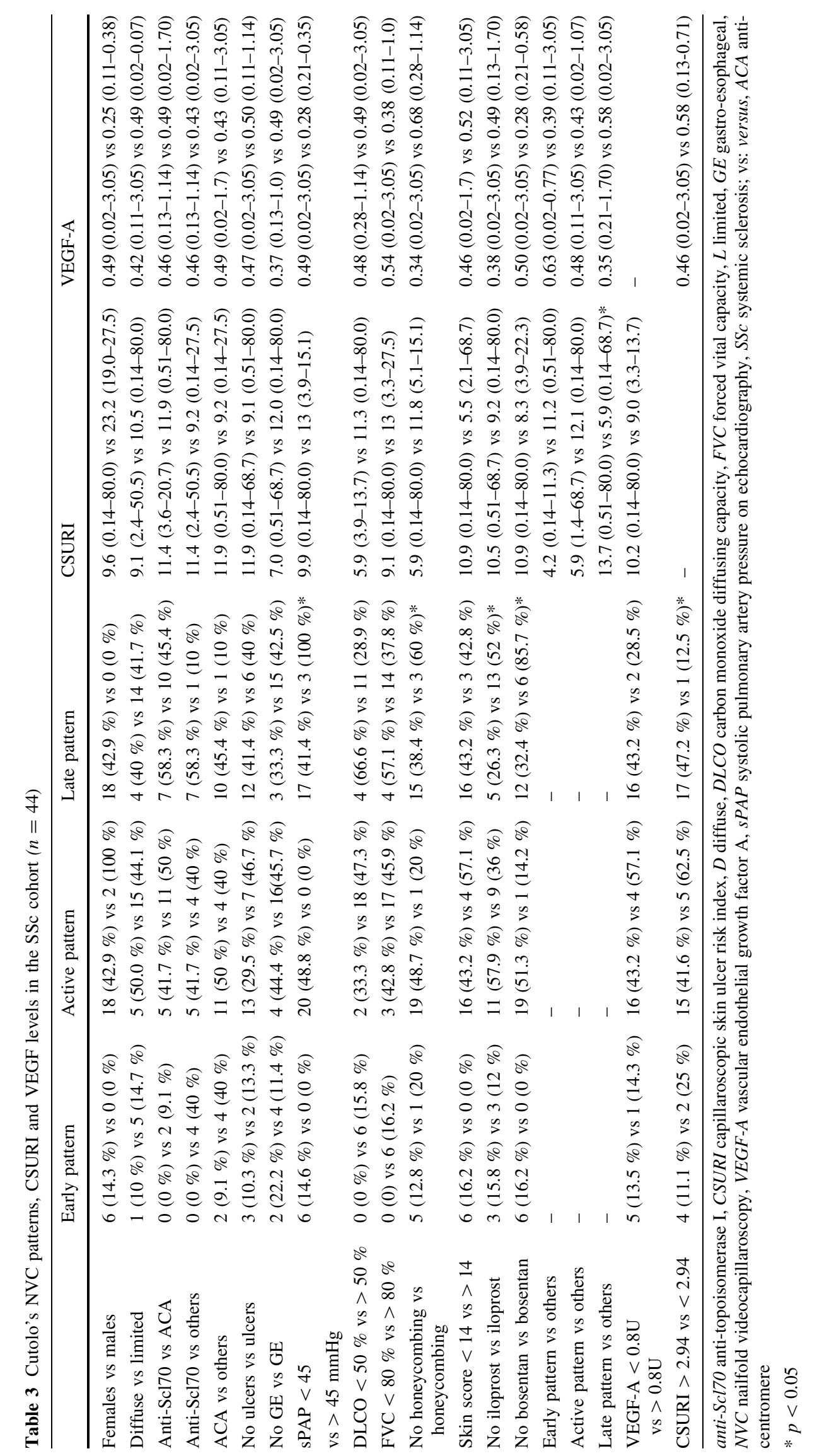



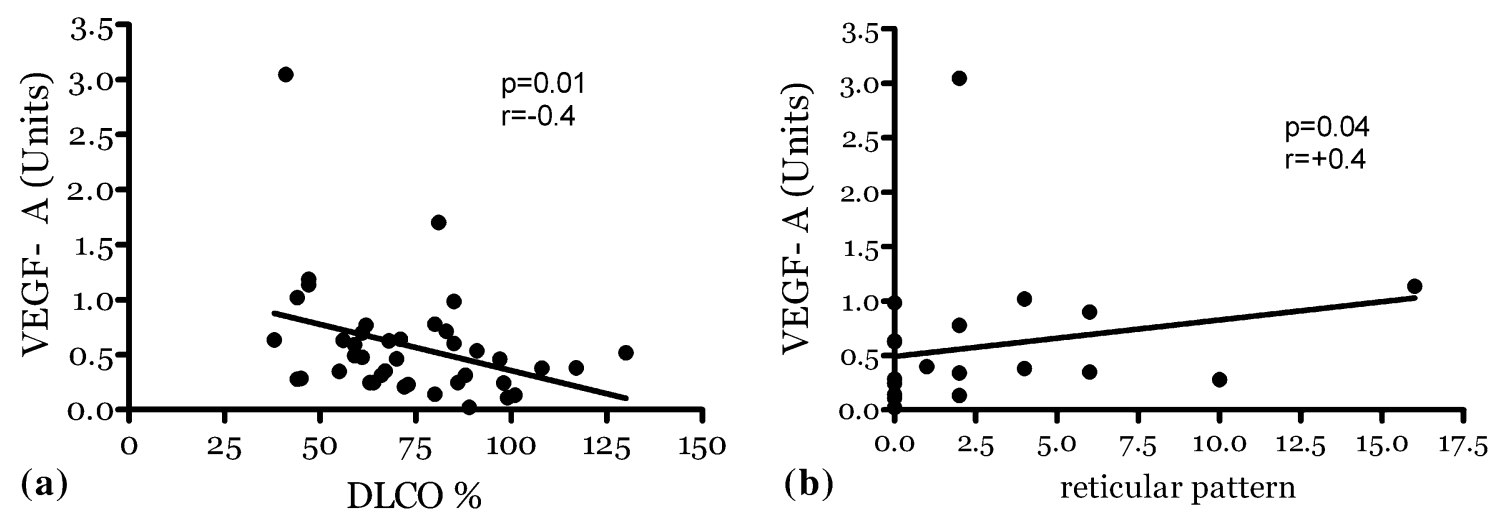

Fig. 4 VEGF-A inversely correlated with DLCO and directly with reticular pattern on high-resolution computed tomography

areas; however, lower SSc disease duration was found in this small group of patients.

Assessing Cutolo's pattern is time sparing, but does not allow a quantitative analysis of microvasculopathy. On the other hand, CSURI is time-consuming and provides limited information. Based on our results, the most helpful and simple data to collect is the mean capillary density. In fact, this parameter not only correlates with an important pulmonary function index (DLCO/AV), but also with SSc skin score, SSc severity index, and with the presence of digital skin ulcers.

The scleroderma NVC pattern and, particularly, the presence of giant capillaries usually predate skin fibrosis as observed in patients reporting with Raynaud's phenomenon. In fact, vasculopathy is thought to precede and possibly cause fibrosis in SSc. The exact mechanism leading to giant capillary development and then to avascular areas appearance is not known. It has been reported that the chronic overexpression of VEGF can lead to enlarged capillary development [25] and that higher levels of VEGF characterize the early phase of SSc when giant capillaries are often the first manifestation of SSc [26]. However, in our study, serum VEGF-A levels did not show any association with NVC alterations. A large study enrolling early SSc is necessary to explore this point, especially when aiming at defining biomarkers for early diagnosis and treatment of SSc [27].

Interstitial lung disease is frequent in SSc patients and in a not well-defined subset of subjects is fatally progressive, being the leading cause of death [28]. We have previously reported lower bronchoalveolar lavage fluid VEGF-A levels in SSc patients with lung involvement, particularly in those with alveolitis on bronchoalveolar lavage fluid analysis and ground-glass on lung HRCT [29]. In healthy adults, an excess of VEGF in the lung compared to other tissues contributes to normal lung tissue homeostasis and endothelial tropism [30]. Nevertheless, in SSc an imbalance between compartments seems to characterize patients with interstitial lung disease, but the pathogenic significance of these data remains to be elucidated. Several reports demonstrated lower VEGF levels in different interstitial lung diseases as lung fibrosis and sarcoidosis [31], but bronchoalveolar lavage fluid analysis is uncomfortable for patients and is not recommended as a routine examination in lung diseases, but in lower airway infection diagnosis [32]. When confirmed in larger SSc cohorts, serum VEGF levels together with pulmonary function tests could be a useful biomarker of interstitial lung disease, maybe better identifying patients needing lung HRCT; moreover, it would be interesting to explore if serum VEGF level changes during the course of SSc, i.e., mirroring lung function decline or predicting progression of SSc interstitial lung disease on HRCT.

\section{Conclusions}

Our data support the importance of NVC not only for the early diagnosis of $\mathrm{SSc}$, but also in the evaluation and possibly prediction of the severity and organ involvement of SSc. We further suggest that prospective studies, ideally multicentric, may confirm the role of serum VEGF levels as putative biomarkers of SSc pulmonary interstitial involvement.

\section{Compliance with ethical standards}

Conflict of interest MDS and AC received travel support from Actelion. The other authors declare that they have no conflict of interest.

Ethical approval All procedures performed in studies involving human participants were in accordance with the ethical standards of the institutional and/or national research committee and with the 1964 Helsinki Declaration and its later amendments or comparable ethical standards.

Informed consent All subjects enrolled signed a written informed consent for serum samples and data analysis after approval from the local ethical committee. 


\section{References}

1. De Santis M, Selmi C (2015) The autoinflammatory side of systemic sclerosis. Isr Med Assoc J 17(1):47-49

2. Selmi C, Feghali-Bostwick CA, Lleo A, Lombardi SA, De Santis M, Cavaciocchi F, Zammataro L, Mitchell MM, Lasalle JM, Medsger T Jr, Gershwin ME (2012) X chromosome gene methylation in peripheral lymphocytes from monozygotic twins discordant for scleroderma. Clin Exp Immunol 169(3):253-262. doi:10.1111/j.1365-2249.2012.04621.x

3. Bogdanos DP, Smyk DS, Rigopoulou EI, Mytilinaiou MG, Heneghan MA, Selmi C, Gershwin ME (2012) Twin studies in autoimmune disease: genetics, gender and environment. $\mathrm{J}$ Autoimmun 38(2-3):J156-J169. doi:10.1016/j.jaut.2011.11.003

4. Selmi C, Invernizzi P, Gershwin ME (2006) The X chromosome and systemic sclerosis. Curr Opin Rheumatol 18(6):601-605. doi:10.1097/01.bor.0000245718.56770.a4

5. Selmi C, Brunetta E, Raimondo MG, Meroni PL (2012) The X chromosome and the sex ratio of autoimmunity. Autoimmun Rev 11(6-7):A531-A537. doi:10.1016/j.autrev.2011.11.024

6. Selmi C, Ceribelli A, Generali E, Scire CA, Alborghetti F, Colloredo G, Porrati L, Achenza MI, De Santis M, Cavaciocchi F, Massarotti M, Isailovic N, Paleari V, Invernizzi P, Matthias T, Zucchi A, Meroni PL (2016) Serum antinuclear and extractable nuclear antigen antibody prevalence and associated morbidity and mortality in the general population over 15 years. Autoimmun Rev 15(2):162-166. doi:10.1016/j.autrev.2015.10. 007

7. van den Hoogen F, Khanna D, Fransen J, Johnson SR, Baron M, Tyndall A, Matucci-Cerinic M, Naden RP, Medsger TA Jr, Carreira PE, Riemekasten G, Clements PJ, Denton CP, Distler O, Allanore Y, Furst DE, Gabrielli A, Mayes MD, van Laar JM, Seibold JR, Czirjak L, Steen VD, Inanc M, Kowal-Bielecka O, Muller-Ladner U, Valentini G, Veale DJ, Vonk MC, Walker UA, Chung L, Collier DH, Csuka ME, Fessler BJ, Guiducci S, Herrick A, Hsu VM, Jimenez S, Kahaleh B, Merkel PA, Sierakowski S, Silver RM, Simms RW, Varga J, Pope JE (2013) 2013 classification criteria for systemic sclerosis: an American College of Rheumatology/European League against Rheumatism collaborative initiative. Arthritis Rheum 65(11):2737-2747. doi:10.1002/ art.38098

8. Cutolo M, Pizzorni C, Tuccio M, Burroni A, Craviotto C, Basso M, Seriolo B, Sulli A (2004) Nailfold videocapillaroscopic patterns and serum autoantibodies in systemic sclerosis. Rheumatology (Oxford) 43(6):719-726. doi:10.1093/rheumatology/ keh156

9. Sebastiani M, Manfredi A, Colaci M, D'Amico R, Malagoli V, Giuggioli D, Ferri C (2009) Capillaroscopic skin ulcer risk index: a new prognostic tool for digital skin ulcer development in systemic sclerosis patients. Arthritis Rheum 61(5):688-694. doi:10. 1002/art.24394

10. Maurer B, Distler A, Suliman YA, Gay RE, Michel BA, Gay S, Distler JH, Distler O (2014) Vascular endothelial growth factor aggravates fibrosis and vasculopathy in experimental models of systemic sclerosis. Ann Rheum Dis 73(10):1880-1887. doi:10. 1136/annrheumdis-2013-203535

11. Manetti M, Guiducci S, Romano E, Ceccarelli C, BellandoRandone S, Conforti ML, Ibba-Manneschi L, Matucci-Cerinic M (2011) Overexpression of VEGF165b, an inhibitory splice variant of vascular endothelial growth factor, leads to insufficient angiogenesis in patients with systemic sclerosis. Circ Res 109(3):e14-e26. doi:10.1161/CIRCRESAHA.111.242057

12. Bielecki M, Kowal K, Lapinska A, Chwiesko-Minarowska S, Chyczewski L, Kowal-Bielecka O (2011) Peripheral blood mononuclear cells from patients with systemic sclerosis spontaneously secrete increased amounts of vascular endothelial growth factor (VEGF) already in the early stage of the disease. Adv Med Sci 56(2):255-263. doi:10.2478/v10039-011-0025-z

13. Kajihara I, Jinnin M, Honda N, Makino K, Makino T, Masuguchi S, Sakai K, Fukushima S, Inoue Y, Ihn H (2013) Scleroderma dermal fibroblasts overexpress vascular endothelial growth factor due to autocrine transforming growth factor beta signaling. Mod Rheumatol 23(3):516-524. doi:10.1007/s10165-012-0698-6

14. Solanilla A, Villeneuve J, Auguste P, Hugues M, Alioum A, Lepreux S, Ducroix JP, Duhaut P, Conri C, Viallard JF, Nurden AT, Constans J, Ripoche J (2009) The transport of high amounts of vascular endothelial growth factor by blood platelets underlines their potential contribution in systemic sclerosis angiogenesis. Rheumatology (Oxford) 48(9):1036-1044. doi:10.1093/ rheumatology/kep154

15. Alivernini S, De Santis M, Tolusso B, Mannocci A, Bosello SL, Peluso G, Pinnelli M, D'Antona G, La Torre G, Ferraccioli G (2009) Skin ulcers in systemic sclerosis: determinants of presence and predictive factors of healing. J Am Acad Dermatol 60(3):426-435. doi:10.1016/j.jaad.2008.11.025

16. Valentini G, Medsger TA Jr, Silman AJ, Bombardieri S (2003) Conclusion and identification of the core set of variables to be used in clinical investigations. Clin Exp Rheumatol 21(3 Suppl 29):S47-S48

17. De Santis M, Bosello S, La Torre G, Capuano A, Tolusso B, Pagliari G, Pistelli R, Danza FM, Zoli A, Ferraccioli G (2005) Functional, radiological and biological markers of alveolitis and infections of the lower respiratory tract in patients with systemic sclerosis. Respir Res 6:96. doi:10.1186/1465-9921-6-96

18. De Santis M, Inzitari R, Bosello SL, Peluso G, Fanali C, Iavarone F, Zizzo G, Bocci M, Cabras T, Messana I, Fuso L, Varone F, Pagliari G, Castagnola M, Ferraccioli G (2011) Beta-thymosins and interstitial lung disease: study of a scleroderma cohort with a 1-year follow-up. Respir Res 12:22. doi:10.1186/1465-9921-1222

19. De Santis M, Bosello SL, Peluso G, Pinnelli M, Alivernini S, Zizzo G, Bocci M, Capacci A, La Torre G, Mannocci A, Pagliari G, Varone F, Pistelli R, Danza FM, Ferraccioli G (2012) Bronchoalveolar lavage fluid and progression of scleroderma interstitial lung disease. Clin Respir J 6(1):9-17. doi:10.1111/j.1752699X.2010.00228.x

20. Yamasaki Y, Narain S, Hernandez L, Barker T, Ikeda K, Segal MS, Richards HB, Chan EK, Reeves WH, Satoh M (2006) Autoantibodies against the replication protein A complex in systemic lupus erythematosus and other autoimmune diseases. Arthritis Res Ther 8(4):R111. doi:10.1186/ar2000

21. Ceribelli A, Cavazzana I, Franceschini F, Airo P, Tincani A, Cattaneo R, Pauley BA, Chan EK, Satoh M (2010) Anti-Th/To are common antinucleolar autoantibodies in Italian patients with scleroderma. J Rheumatol 37(10):2071-2075. doi:10.3899/ jrheum. 100316

22. Cavazzana I, Fredi M, Selmi C, Tincani A, Franceschini F (2015) The clinical and histological spectrum of idiopathic inflammatory myopathies. Clin Rev Allergy Immunol. doi:10.1007/s12016015-8517-4

23. Cavagna L, Nuno L, Scire CA, Govoni M, Longo FJ, Franceschini F, Neri R, Castaneda S, Sifuentes Giraldo WA, Caporali R, Iannone F, Fusaro E, Paolazzi G, Pellerito R, Schwarting A, Saketkoo LA, Ortego-Centeno N, Quartuccio L, Bartoloni E, Specker C, Pina Murcia T, La Corte R, Furini F, Foschi V, Bachiller Corral J, Airo P, Cavazzana I, Martinez-Barrio J, Hinojosa M, Giannini M, Barsotti S, Menke J, Triantafyllias K, Vitetta R, Russo A, Bogliolo L, Bajocchi G, Bravi E, Barausse G, Bortolotti R, Selmi C, Parisi S, Salaffi F, Montecucco C, Gonzalez-Gay MA, Group AC (2016) Serum Jo-1 autoantibody and isolated arthritis in the antisynthetase syndrome: review of the 
literature and report of the experience of AENEAS Collaborative Group. Clin Rev Allergy Immunol. doi:10.1007/s12016-0168528-9

24. Cavagna L, Nuno L, Scire CA, Govoni M, Longo FJ, Franceschini F, Neri R, Castaneda S, Giraldo WA, Caporali R, Iannone F, Fusaro E, Paolazzi G, Pellerito R, Schwarting A, Saketkoo LA, Ortego-Centeno N, Quartuccio L, Bartoloni E, Specker C, Murcia TP, La Corte R, Furini F, Foschi V, Corral JB, Airo P, Cavazzana I, Martinez-Barrio J, Hinojosa M, Giannini M, Barsotti S, Menke J, Triantafyllias K, Vitetta R, Russo A, Bajocchi G, Bravi E, Barausse G, Bortolotti R, Selmi C, Parisi S, Montecucco C, Gonzalez-Gay MA, group Ac (2015) Clinical spectrum time course in anti Jo-1 positive antisynthetase syndrome: results from an International Retrospective Multicenter Study. Medicine (Baltimore) 94(32):e1144. doi:10.1097/MD.0000000000001144

25. Karvinen H, Pasanen E, Rissanen TT, Korpisalo P, Vahakangas E, Jazwa A, Giacca M, Yla-Herttuala S (2011) Long-term VEGFA expression promotes aberrant angiogenesis and fibrosis in skeletal muscle. Gene Ther 18(12):1166-1172. doi:10.1038/gt. 2011.66

26. Distler O, Del Rosso A, Giacomelli R, Cipriani P, Conforti ML, Guiducci S, Gay RE, Michel BA, Bruhlmann P, Muller-Ladner U, Gay S, Matucci-Cerinic M (2002) Angiogenic and angiostatic factors in systemic sclerosis: increased levels of vascular endothelial growth factor are a feature of the earliest disease stages and are associated with the absence of fingertip ulcers. Arthritis Res 4(6):R11
27. Bournia VK, Vlachoyiannopoulos PG, Selmi C, Moutsopoulos HM, Gershwin ME (2009) Recent advances in the treatment of systemic sclerosis. Clin Rev Allergy Immunol 36(2-3):176-200. doi:10.1007/s12016-008-8114-x

28. Bosello S, De Santis M, Lama G, Spano C, Angelucci C, Tolusso B, Sica G, Ferraccioli G (2010) B cell depletion in diffuse progressive systemic sclerosis: safety, skin score modification and IL-6 modulation in an up to thirty-6 months follow-up open-label trial. Arthritis Res Ther 12(2):R54. doi:10.1186/ar2965

29. De Santis M, Bosello SL, Capoluongo E, Inzitari R, Peluso G, Lulli P, Zizzo G, Bocci M, Tolusso B, Zuppi C, Castagnola M, Ferraccioli G (2012) A vascular endothelial growth factor deficiency characterises scleroderma lung disease. Ann Rheum Dis 71(9):1461-1465. doi:10.1136/annrheumdis-2011-200657

30. Kaner RJ, Crystal RG (2001) Compartmentalization of vascular endothelial growth factor to the epithelial surface of the human lung. Mol Med 7(4):240-246

31. Meyer KC, Cardoni A, Xiang ZZ (2000) Vascular endothelial growth factor in bronchoalveolar lavage from normal subjects and patients with diffuse parenchymal lung disease. J Lab Clin Med 135(4):332-338. doi:10.1067/mlc.2000.105618

32. De Franceschi L, Bosello S, Scambi C, Biasi D, De Santis M, Caramaschi P, Peluso G, La Verde V, Bambara LM, Ferraccioli G (2011) Proteome analysis of biological fluids from autoimmune-rheumatological disorders. Proteom Clin Appl 5(1-2):78-89. doi:10.1002/prca.201000069 